\title{
The $\Sigma-D$ analysis of recently detected radio planetary nebulae in the Magellanic Clouds (Research Note)
}

\author{
B. Vukotić ${ }^{1}$, D. Urošević ${ }^{2,4}$, M. D. Filipović ${ }^{3}$, and J. L. Payne ${ }^{3}$
}

\author{
1 Astronomical Observatory Belgrade, Volgina 7, 11160 Belgrade-74, Serbia \\ e-mail: bvukotic@aob.bg.ac.yu \\ 2 University of Belgrade, Faculty of Mathematics, Studentski trg 16, 11000 Belgrade, Serbia \\ e-mail: dejanu@matf.bg.ac.yu \\ 3 University of Western Sydney, Locked Bag 1797, Penrith South, DC, NSW 1797, Australia \\ e-mail:m.filipovic@uws.edu.au, snova4@msn.com \\ 4 Isaac Newton Institute of Chile, Yugoslavia Branch, Yugoslavia
}

Received 6 February 2009 / Accepted 26 June 2009

\section{ABSTRACT}

\begin{abstract}
Aims. We investigate and analyze the radio surface brightness to diameter $(\Sigma-D)$ relation for recently detected, bright radio-continuum planetary nebulae (PNe) in the Magellanic Clouds (MC).

Methods. We applied a Monte Carlo analysis in order to account for sensitivity selection effects on measured $\Sigma-D$ relation slopes for bright radio PNe in the MCs.

Results. In the $\Sigma-D$ plane, these radio MCs PNe are positioned among the brightest of the nearby Galactic PNe and are close to the $D^{-2}$ sensitivity line of the MCs radio maps. The fitted Large Magellanic Cloud (LMC) data slope appears to be influenced by survey sensitivity. This suggests the MCs radio PN sample represents just the "tip of the iceberg" of the actual luminosity function. Specifically, our results imply that sensitivity selection tends to flatten the slope of the $\Sigma-D$ relation. Although MCs PNe appear to share the evolution properties of Galactic PNe, the small number of data points prevented us from further constraining their evolution properties.
\end{abstract}

Key words. methods: statistical - ISM: planetary nebulae: general - galaxies: Magellanic Clouds - radio continuum: ISM

\section{Introduction}

Filipović et al. (2009) has recently reported 15 radio-continuum $\mathrm{PNe}$ detected at the $3 \sigma$ level and above from various radio Magellanic Clouds (MCs) surveys. Their detections are mainly based on positional coincidences with optically detected planetary nebulae (PNe) and optical spectroscopy (Payne et al. 2008a,b). Included are Large Magellanic Cloud (LMC) mosaics (Dickel et al. 2005) with sensitivities of $\sim 0.5 \mathrm{mJy}^{\text {beam }}{ }^{-1}$ at both 4.8 and $8.64 \mathrm{GHz}$ with resolutions of 33 and 20", respectively. The Small Magellanic Cloud (SMC) mosaics have $0.5 \mathrm{mJy}$ beam $^{-1}$ sensitivities at 4.8 and $8.64 \mathrm{GHz}$ with resolutions of 30 and 15" (Dickel 2009). To compare these sources with Galactic PNe, we use radio surface brightness $(\Sigma)$ since this quantity is distance independent:

$\Sigma\left[\mathrm{Wm}^{-2} \mathrm{~Hz}^{-1} \mathrm{sr}^{-1}\right]=1.505 \times 10^{-19} \frac{S[\mathrm{Jy}]}{\theta^{2}\left[{ }^{\prime}\right]}$,

where $S$ is the flux density and $\theta$, the angular diameter of the source. Theory predicts the existence of a linear relation between $\log \Sigma$ and $\log D$, with $D$ representing the diameter of the object (see Urosevic et al. 2007; Urošević et al. 2009, and references therein).

All of the detected radio $\mathrm{PNe}$ are unresolved, despite five of them being observed in "snap-shot" mode with the resolution of up to $1^{\prime \prime}$ and sensitivity of $\sim 0.1 \mathrm{mJy}^{\text {beam }}{ }^{-1}$ (for details see Filipović et al. 2009). The optical diameters are available for some 10 out of 15 (see Table 1 in Filipović et al. 2009). The MCs $\mathrm{PNe}$ data at $4.8 \mathrm{GHz}$ are plotted in Fig. 1 with respective LMC map $3 \sigma$ sensitivity line at $1.5 \mathrm{mJy}^{-1}$ beam $^{-1}$ (hereafter referred to as sensitivity line). Five LMC PNe are above the sensitivity line and one is below ("snap-shot" mode). Five PNe with estimated upper flux density limit of $1.5 \mathrm{mJy}^{\text {beam }}{ }^{-1}$ are plotted on the sensitivity line and the remaining one without optical diameter is not plotted. Four SMC PNe are plotted on the SMC map resolution limit of $30^{\prime \prime}$. We use the PNe sample of Galactic PNe with reliable individual distances from Stanghellini et al. (2008; hereafter SSV), and divide them into two subsamples (nearby PNe with distances smaller than $1 \mathrm{kpc}$ and those with distances greater than $1 \mathrm{kpc}$ ). Urošević et al. (2009), in an extensive analysis of the empirical $\Sigma-D$ relation for PNe, argued that the SSV sample of nearby PNe is least influenced by selection effects. For comparison, we plot two radio $\mathrm{PNe}$ from the Sagittarius dwarf galaxy (Dudziak et al. 2000), also representative of extragalactic radio $\mathrm{PNe}$. An arrow in Fig. 1 represents the $D^{-2}$ direction in which unresolved objects should move if their diameters were known.

Inspection of Fig. 1 reveal that the detected radio-continuum MCs PNe are very close to their sensitivity lines and they are as bright as the brightest Galactic PNe. When compared with Galactic PNe it appears that current data set is only the very peak of the actual MCs PNe luminosity function. In this paper, using $\chi^{2}$ analysis and Monte Carlo generated data samples, we 


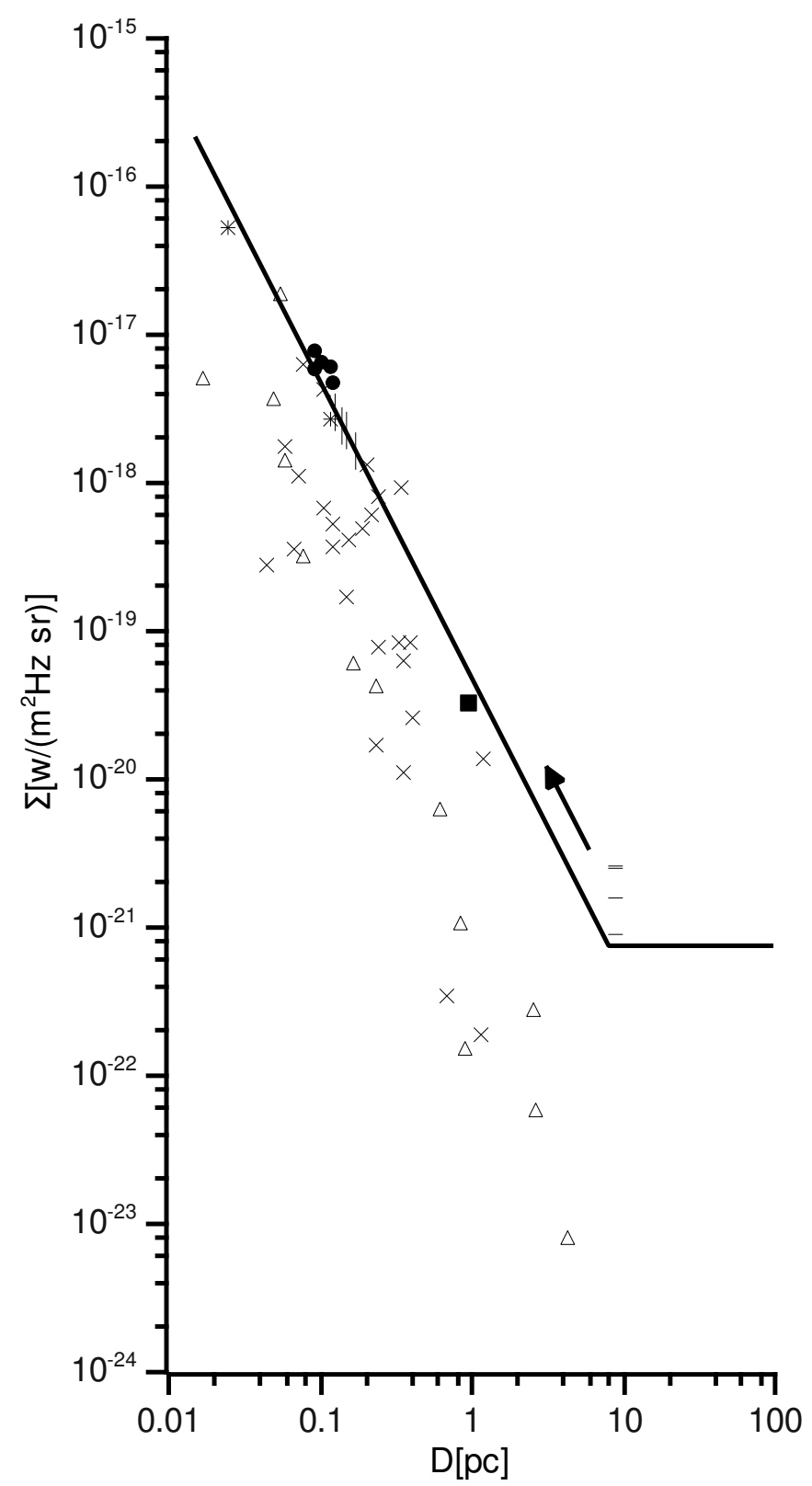

Fig. 1. A plot of $4.8 \mathrm{GHz}$ PNe from the LMC, SMC and Sagittarius Dwarf Galaxy. The solid line represents the LMC map sensitivity $(3 \sigma)$ at $1.5 \mathrm{mJy} \mathrm{beam}^{-1}$. The LMC radio PNe with known optical diameters are represented with filled circles (above the sensitivity line, $5 \mathrm{PNe}$ ) and vertical dashes (sensitivity line is the upper flux density limit, $5 \mathrm{PNe}$ ). One LMC PN observed in "snap-shot" mode is represented with filled square. The SMC radio PNe without known optical diameters are represented by horizontal dashes and asterisks are two Sagittarius Dwarf PNe. Open triangles represents Galactic SSV PNe with distances greater than $1 \mathrm{kpc}$ while diagonal crosses are those with distances less than $1 \mathrm{kpc}$. Note that the arrow represents the direction in which MCs radio $\mathrm{PNe}$ should move on the graph, if their diameters were known.

investigate the sensitivity related selection effects on the $\Sigma-D$ properties of these objects and compare them with the properties of Galactic PNe.

As a convenient platform for distance determination, the $\Sigma-D$ relation ${ }^{1}$ has a $\sim 50$ year long history of exploitation for supernova remnants (SNRs). The study of the relation for these very luminous radio synchrotron sources began with the work of

${ }^{1}$ In this paper, we use the form $\Sigma \propto D^{\beta}$, where $\beta$ represents slope.
Shklovskii (1960), while the first empirical $\Sigma-D$ relation was presented by Poveda \& Woltjer (1968). Decades of analysis resulted in a good understanding of the $\Sigma-D$ relation for SNRs and various data sample selection effects that influence that relation.

The previous analysis of PNe radio data samples (e.g. Phillips 2002, and references therein) were mainly focused on radius vs radio brightness temperature $\left(R-T_{\mathrm{b}}\right)$ empirical relations. These were sparse in the consideration of data selection effects. Recent works by Urosevic et al. (2007) and Urošević et al. (2009) use a SNR formalism in the analysis of PNe data along with a discussion of these selection effects. Although they note that all PN samples suffer from selection effects caused by limitations in survey sensitivity and resolution, the nearby SSV sample, having a $\Sigma-D$ slope of $\beta=-2.61 \pm 0.21$, is least influenced by these effects.

\section{Analysis}

Samples for Monte Carlo simulations are made using sources from Table 1 in Filipović et al. (2009) that have a known optical diameter (LMC sources only) and are above the map sensitivity line for a given frequency. This includes $5 \mathrm{PNe}$ at both frequencies $(8.64$ and $4.8 \mathrm{GHz}$ ) though not necessarily the same ones. Using adopted distances to the LMC of $50 \mathrm{kpc}$ and to the SMC of $60 \mathrm{kpc}$ (Alves 2004; Hilditch et al. 2005), the smallest sources in the selected samples have a mean geometrical diameter of $0.09 \mathrm{pc}$ at both frequencies. The largest source has a mean geometrical diameter of $0.12 \mathrm{pc}$ at $4.8 \mathrm{GHz}$ and $0.15 \mathrm{pc}$ at $8.64 \mathrm{GHz}$. Surface brightness ranges from $2.67 \times 10^{-18}$ to $8.65 \times$ $10^{-18} \mathrm{Wm}^{-2} \mathrm{~Hz}^{-1} \mathrm{sr}^{-1}$ at $8.64 \mathrm{GHz}$ and from $4.70 \times 10^{-18}$ to $7.90 \times 10^{-18} \mathrm{Wm}^{-2} \mathrm{~Hz}^{-1} \mathrm{sr}^{-1}$ at $4.8 \mathrm{GHz}$. For the LMC maps, the 4.8 GHz sensitivity line is at $\Sigma=7.46 \times 10^{-22} \mathrm{Wm}^{-2} \mathrm{~Hz}^{-1} \mathrm{sr}^{-1}$ with a $D^{-2}$ break at $D=8.00 \mathrm{pc}$ and a $8.64 \mathrm{GHz}$ sensitivity line at $\Sigma=2.03 \times 10^{-21}$ and $D=4.85 \mathrm{pc}$, respectively.

The relative flux density errors at both frequencies of the LMC maps are $<10 \%$ (Filipović et al. 2009) and they are approximately the size of the symbols shown in Fig. 1. If we assume that the optical diameters have no significant errors and that the absolute flux density error equals the flux density standard deviation $\sigma_{\mathrm{S}}$, according to error propagation theory it follows from Eq. (1). that the standard deviation for $\log \Sigma\left(\sigma_{\log \Sigma}\right)$ is $0.434 \Delta S / S$. This gives $\sigma_{\log \Sigma} \approx 0.05$.

The resulting parameters of the $\log \Sigma=a+\beta * \log D$ fits are given in Table 1 for each frequency. The parameters $a$ and $\beta$, their standard uncertainties $\Delta a$ and $\Delta \beta$, the linear correlation coefficient $r$, the fit quality $r^{2}$, the probability $Q$ of obtaining larger WSSR (weighted sum of square residuals) and a ratio of WSSR to the number of degrees of freedom (ndof) are given in this table. A value of $0.1 \gtrsim Q \gtrsim 0.001$ is expected for a statistically acceptable fit (the case when errors of the dependent variable have a non-Gaussian distribution), if the data is were well approximated by the model. This should apply for fits presented in this paper since we have transformed flux density errors to $\log \Sigma$ errors. When introduced in the least squares fitting procedure, our values of $Q$ implies a statistically acceptable fit.

Although the fits are statistically believable the resulting $\beta$ at $4.8 \mathrm{GHz}$ substantially differs from $\beta$ at $8.64 \mathrm{GHz}$, likely in debt to a small number of data points. Sampling only the peak of the luminosity function and with the majority of the sample likely hidden below the sensitivity line, the resulting values for $\beta$ should not be taken seriously. Comparing the LMC radio PN sample and the sample of a nearby Galactic PNe, we made an 
Table 1. Fit parameters of the LMC data sample.

\begin{tabular}{ccccccccc}
\hline \hline Frequency & $a$ & $\Delta a$ & $\beta$ & $\Delta \beta$ & $r$ & $r^{2}[\%]$ & $Q$ & $\frac{\text { WSSR }}{\text { ndof }}$ \\
\hline $8.64 \mathrm{GHz}$ & -19.7 & 0.3 & -2.6 & 0.4 & -0.94 & 88.36 & $5.02 \times 10^{-2}$ & 2.60 \\
$4.80 \mathrm{GHz}$ & -18.1 & 0.5 & -0.9 & 0.5 & -0.64 & 40.96 & $9.96 \times 10^{-2}$ & 2.08 \\
\hline
\end{tabular}

attempt to access a more meaningful $\Sigma-D$ slope using Monte Carlo simulations as described below.

We performed Monte Carlo simulations similar to those described by Urošević et al. (2005). The results of their simulations (with 21 data points above the sensitivity line) showed that slopes steeper than -2 are under influence of sensitivity related selection effects. First, we determined the empirical $\log \Sigma$ standard deviation from the best fit line, assuming $\log D$ as the independent variable. We then selected an interval in $\log D$ five times as long as that of the real data. This interval is then sprinkled with random points of the same $\log D$ density as that of the real data.

The simulated points, that lie on the $\log \mathrm{D}$ axis, are then projected onto a series of lines at different slopes (in steps of 0.1 from -3.5 to -1.5 ). Each of these lines passes through the extreme upper left hand end of the best fit line to the real data. We also added Gaussian noise in $\log \Sigma$, which is related to the scatter of the real data by a parameter called "scatter". A scatter of 1 corresponds to the same standard deviation as that of the real data.

An appropriate sensitivity cutoff is applied to the simulated data points, selecting points above the sensitivity line. This is done 100 times for each simulated slope and a least squares best fit line is generated for artificial samples that have five or more selected data points. The number of such samples is given in the last column of Tables 2 and 3 .

In Tables 2 and 3, the first column lists the value of the simulated slope, while the mean and standard deviation of the best fit slopes for the generated samples are given in second and third column, respectively. The fourth and fifth column gives the mean and standard deviation of the best fit slopes for sensitivity selected generated samples, respectively. In Fig. 2 we present one of our Monte Carlo generated samples at $8.64 \mathrm{GHz}$ for a scatter of 1 and the simulated slope -2.7 .

\section{Discussion and conclusions}

From Tables 2 and 3, it is evident that the sensitivity cutoff tends to flatten the intrinsic (simulated) slopes less than -2 (the most likely case for the real data). At $4.8 \mathrm{GHz}$, a slope of $-0.9 \pm 0.5$ (shallower than the sensitivity line) is not influenced by sensitivity related selection effects. From the other side, this slope does not have any physical interpretation and is only the consequence of scatter in the $\Sigma-D$ plane. The $8.64 \mathrm{GHz}$ slope of $-2.6 \pm 0.4$ appears to correspond to somewhat steeper selection free slopes but due to a large error could have any value smaller than -2.3. This is within the range of the SSV slope at $4.8 \mathrm{GHz}$. With current LMC data samples it is not possible to constrain the lower limit, due to a smaller number of selected points for steeper slopes. The value of a mean slope after selection at $8.64 \mathrm{GHz}$ starts to oscillate for the slopes $\lesssim-2.4$ (the standard deviation of slope after selection exceeds the interval between two successive simulated slopes). Table 2 is given here rather for completeness, but nevertheless shows that for the samples with small number of data points and a slope greater than
Table 2. Monte Carlo simulation results at $4.8 \mathrm{GHz}$.

\begin{tabular}{|c|c|c|c|c|c|}
\hline $\begin{array}{l}\text { The slope } \\
\text { that is } \\
\text { simulated }\end{array}$ & $\begin{array}{c}\text { Mean } \\
\text { simulated } \\
\text { slope }\end{array}$ & $\begin{array}{l}\text { Standard } \\
\text { deviation } \\
\text { of mean } \\
\text { simulated } \\
\text { slope }\end{array}$ & $\begin{array}{c}\text { Mean } \\
\text { slope } \\
\text { after } \\
\text { selection }\end{array}$ & $\begin{array}{c}\text { Standard } \\
\text { deviation } \\
\text { of slope } \\
\text { after } \\
\text { selection }\end{array}$ & $\begin{array}{c}\text { No. of } \\
\text { generated } \\
\text { samples } \\
\text { that was } \\
\text { fitted }\end{array}$ \\
\hline \multicolumn{6}{|c|}{ scatter $=1.0$} \\
\hline-1.500000 & -1.492251 & 0.068066 & -1.514832 & 0.064286 & 100 \\
\hline-1.600000 & -1.598043 & 0.068590 & -1.623227 & 0.062590 & 100 \\
\hline-1.700000 & -1.699330 & 0.065987 & -1.728391 & 0.064682 & 100 \\
\hline-1.800000 & -1.801370 & 0.061776 & -1.827670 & 0.062875 & 100 \\
\hline-1.900000 & -1.904920 & 0.065998 & -1.939695 & 0.063711 & 100 \\
\hline-2.000000 & -2.003822 & 0.057447 & -2.001172 & 0.059317 & 100 \\
\hline-2.100000 & -2.099951 & 0.065622 & -2.041389 & 0.062283 & 100 \\
\hline-2.200000 & -2.195110 & 0.063386 & -2.070956 & 0.086876 & 99 \\
\hline-2.300000 & -2.297590 & 0.064135 & -2.074784 & 0.130676 & 82 \\
\hline-2.400000 & -2.398137 & 0.070116 & -2.110495 & 0.211172 & 59 \\
\hline-2.500000 & -2.501291 & 0.065666 & -2.180770 & 0.426777 & 40 \\
\hline-2.600000 & -2.594927 & 0.068783 & -2.222386 & 0.527765 & 23 \\
\hline-2.700000 & -2.700259 & 0.065226 & -2.347649 & 0.527067 & 11 \\
\hline-2.800000 & -2.801124 & 0.071673 & -2.339542 & 0.345267 & 11 \\
\hline-2.900000 & -2.892171 & 0.061980 & -2.325770 & 0.384151 & 9 \\
\hline-3.000000 & -2.995751 & 0.067793 & -2.814787 & 0.608717 & 4 \\
\hline-3.100000 & -3.093523 & 0.066956 & -2.789218 & 0.225177 & 3 \\
\hline-3.200000 & -3.093523 & 0.131417 & - & - & 0 \\
\hline-3.300000 & -3.304876 & 0.071690 & -2.841456 & 0.077769 & 3 \\
\hline-3.400000 & -3.304876 & 0.126657 & -2.841456 & - & 1 \\
\hline-3.500000 & -3.502017 & 0.064626 & -2.471982 & 0.471662 & 3 \\
\hline \multicolumn{6}{|c|}{ scatter $=2.0$} \\
\hline-1.500000 & -1.494408 & 0.129362 & -1.582530 & 0.129317 & 100 \\
\hline-1.600000 & -1.582302 & 0.137065 & -1.684048 & 0.127792 & 100 \\
\hline-1.700000 & -1.710318 & 0.142266 & -1.786533 & 0.126412 & 100 \\
\hline-1.800000 & -1.803957 & 0.135674 & -1.896290 & 0.127258 & 100 \\
\hline-1.900000 & -1.908844 & 0.128375 & -1.957930 & 0.123818 & 100 \\
\hline-2.000000 & -1.977503 & 0.128244 & -1.979623 & 0.101482 & 100 \\
\hline-2.100000 & -2.126453 & 0.124008 & -2.047942 & 0.106982 & 100 \\
\hline-2.200000 & -2.218740 & 0.132994 & -2.069085 & 0.121897 & 99 \\
\hline-2.300000 & -2.301067 & 0.118757 & -2.100132 & 0.180063 & 96 \\
\hline-2.400000 & -2.422463 & 0.156185 & -2.121199 & 0.220895 & 85 \\
\hline-2.500000 & -2.489904 & 0.122634 & -2.137602 & 0.234918 & 73 \\
\hline-2.600000 & -2.596131 & 0.130540 & -2.133221 & 0.314863 & 54 \\
\hline-2.700000 & -2.715585 & 0.105664 & -2.166716 & 0.624909 & 38 \\
\hline-2.800000 & -2.784944 & 0.118933 & -2.127692 & 0.398607 & 30 \\
\hline-2.900000 & -2.922056 & 0.123773 & -2.334104 & 0.607681 & 16 \\
\hline-3.000000 & -3.007808 & 0.115488 & -2.198724 & 1.185409 & 20 \\
\hline-3.100000 & -3.090180 & 0.137786 & -2.386109 & 0.311125 & 10 \\
\hline-3.200000 & -3.181103 & 0.140162 & -2.454902 & 1.599802 & 6 \\
\hline-3.300000 & -3.319871 & 0.139671 & -1.996534 & 0.496468 & 9 \\
\hline-3.400000 & -3.389912 & 0.124211 & -2.349205 & 0.254864 & 4 \\
\hline-3.500000 & -3.389912 & 0.168488 & -2.349205 & - & 1 \\
\hline
\end{tabular}

-2 it is not possible to extract any meaningful information with this kind of simulations.

With these recently confirmed MCs radio $\mathrm{PNe}$ being amongst brightest Galactic PNe in the $\Sigma-D$ plane and given their proximity to the sensitivity line, it is likely they represent only the peak of the actual MCs PNe luminosity function, which is probably the "bright-end" extension of the Galactic PNe luminosity function (for a more detailed discussion of the nature of these MCs PNe see Filipović et al. 2009). The results of our LMC Monte Carlo simulations suggest that current sparse data samples cannot give meaningful $\Sigma-D$ slope. However, they 
Table 3. Monte Carlo simulation results at $8.64 \mathrm{GHz}$.

\begin{tabular}{|c|c|c|c|c|c|}
\hline $\begin{array}{l}\text { The slope } \\
\text { that is } \\
\text { simulated }\end{array}$ & $\begin{array}{c}\text { Mean } \\
\text { simulated } \\
\text { slope }\end{array}$ & $\begin{array}{l}\text { Standard } \\
\text { deviation } \\
\text { of mean } \\
\text { simulated } \\
\text { slope }\end{array}$ & $\begin{array}{c}\text { Mean } \\
\text { slope } \\
\text { after } \\
\text { selection }\end{array}$ & $\begin{array}{c}\text { Standard } \\
\text { deviation } \\
\text { of slope } \\
\text { after } \\
\text { selection }\end{array}$ & $\begin{array}{c}\text { No. of } \\
\text { generated } \\
\text { samples } \\
\text { that was } \\
\text { fitted }\end{array}$ \\
\hline \multicolumn{6}{|c|}{ scatter $=1.0$} \\
\hline-1.500000 & -1.503405 & 0.052604 & -1.503405 & 0.052604 & 100 \\
\hline-1.600000 & -1.600591 & 0.042812 & -1.601002 & 0.042035 & 100 \\
\hline-1.700000 & -1.698601 & 0.037607 & -1.698601 & 0.037607 & 100 \\
\hline-1.800000 & -1.792767 & 0.040580 & -1.792767 & 0.040580 & 100 \\
\hline-1.900000 & -1.893138 & 0.043315 & -1.893138 & 0.043315 & 100 \\
\hline-2.000000 & -1.995468 & 0.045243 & -1.994920 & 0.045646 & 100 \\
\hline-2.100000 & -2.102842 & 0.042350 & -2.088784 & 5885 & 100 \\
\hline-2.200000 & -2.193731 & 0.042313 & -2.144698 & 331 & 100 \\
\hline-2.300000 & -2.298443 & 0.045962 & -2.187980 & 0.069080 & 100 \\
\hline-2.400000 & -2.401427 & 0.048780 & -2.269207 & 0.107564 & 100 \\
\hline-2.500000 & -2.501493 & 0.0 & -2.318480 & 2147 & 98 \\
\hline-2.600000 & -2.601538 & 0.04 & -2.425642 & 0.195200 & 98 \\
\hline-2.700000 & -2.699132 & 0.04 & -2.449996 & 0.249949 & 81 \\
\hline-2.800000 & -2.803434 & 787 & -2.553920 & 8122 & 62 \\
\hline-2.900000 & -2.895824 & 305 & -2.556149 & 9145 & 56 \\
\hline-3.000000 & -3.000702 & 0.041717 & -2.694128 & 0.30 & 49 \\
\hline-3.100000 & -3.100702 & 0.03 & -2.703438 & 0.55 & 40 \\
\hline-3.200000 & -3.199087 & 0.04 & -2.836055 & 9287 & 28 \\
\hline-3.300000 & -3.303262 & 0.042204 & -2.874302 & 416 & 15 \\
\hline-3.400000 & & 0.0 & -2.775834 & 0.4 & 18 \\
\hline-3.500000 & -3.4 & 0.0 & -2.9 & 0.4 & 14 \\
\hline \multicolumn{6}{|c|}{ scatter $=2.0$} \\
\hline-1.500000 & -1.49 & 0.0 & -1.506813 & 878 & 100 \\
\hline-1.600000 & -1.595906 & 0.0 & -1.609181 & 654 & 100 \\
\hline-1.700000 & -1.706313 & 0.079195 & -1.720427 & 0.077824 & 100 \\
\hline-1.800000 & -1.801306 & 0.077013 & -1.818025 & 0.079255 & 100 \\
\hline-1.900000 & -1.89 & & -1. & 89 & 100 \\
\hline-2.000000 & -2.002529 & 0.093860 & -2.007141 & 0.092602 & 100 \\
\hline-2.100000 & -2.094921 & 0.087675 & -2.065955 & 0.079765 & 100 \\
\hline-2.200000 & -2.196983 & 0.09 & -2.109052 & 0.089702 & 100 \\
\hline-2.300000 & -2.278 & 11 & -2.135513 & 328 & 100 \\
\hline-2.400000 & -2.403615 & 0.089715 & -2.205754 & 0.141621 & 100 \\
\hline-2.500000 & -2.478826 & 0.080478 & -2.194225 & 0.191552 & 98 \\
\hline-2.600000 & -2.593142 & 0.095069 & -2.264253 & 0.276645 & 89 \\
\hline-2.700000 & -2.709181 & 0.091667 & -2.402365 & 0.329761 & 85 \\
\hline-2.800000 & -2.799243 & 0.076143 & -2.297942 & 0.374669 & 67 \\
\hline-2.900000 & -2.901775 & 0.080564 & -2.406221 & 0.411391 & 61 \\
\hline-3.000000 & -2.999507 & 0.086134 & -2.591900 & 0.528155 & 41 \\
\hline-3.100000 & -3.083982 & 0.090140 & -2.527112 & 0.497492 & 31 \\
\hline-3.200000 & -3.187697 & 0.081336 & -2.453453 & 0.617842 & 29 \\
\hline-3.300000 & -3.303730 & 9221 & -2.681570 & 1.138125 & 32 \\
\hline-3.400000 & -3.392252 & 0.084091 & -2.712006 & 0.649541 & 20 \\
\hline-3.500000 & -3.501420 & 0.078870 & -2.584415 & 0.885438 & 21 \\
\hline
\end{tabular}

confirmed that the corresponding apparent $\Sigma-D$ slope flattens toward $\beta \approx-2$ because of the sensitivity related selection effects..

This should be kept in mind as a serious measure of caution in forthcoming studies of extragalactic PN samples. Future high sensitivity images of the MCs will surely provide even better and more complete radio samples of PN population and enable more robust constraining of $\mathrm{PN}$ evolution parameters.

Acknowledgements. The authors would like to thank the referee Prof. John Dickel for useful comments that have improved the manuscript. This research has been supported by the Ministry of Science and Technological Development of the Republic of Serbia through the Project \#146012.

\section{References}

Alves, D. R. 2004, New Astron. Rev. 48, 659

Dickel, J. R., McIntyre, V. J., Gruendl, R. A., \& Milne, D. K. 2005, AJ, 129, 790 Dickel, J. R., et al. 2009, in The Magellanic System: Stars, Gas, and Galaxies, ed. J. van Loon, \& J. M. Oliveira (Cambridge University Press), in press

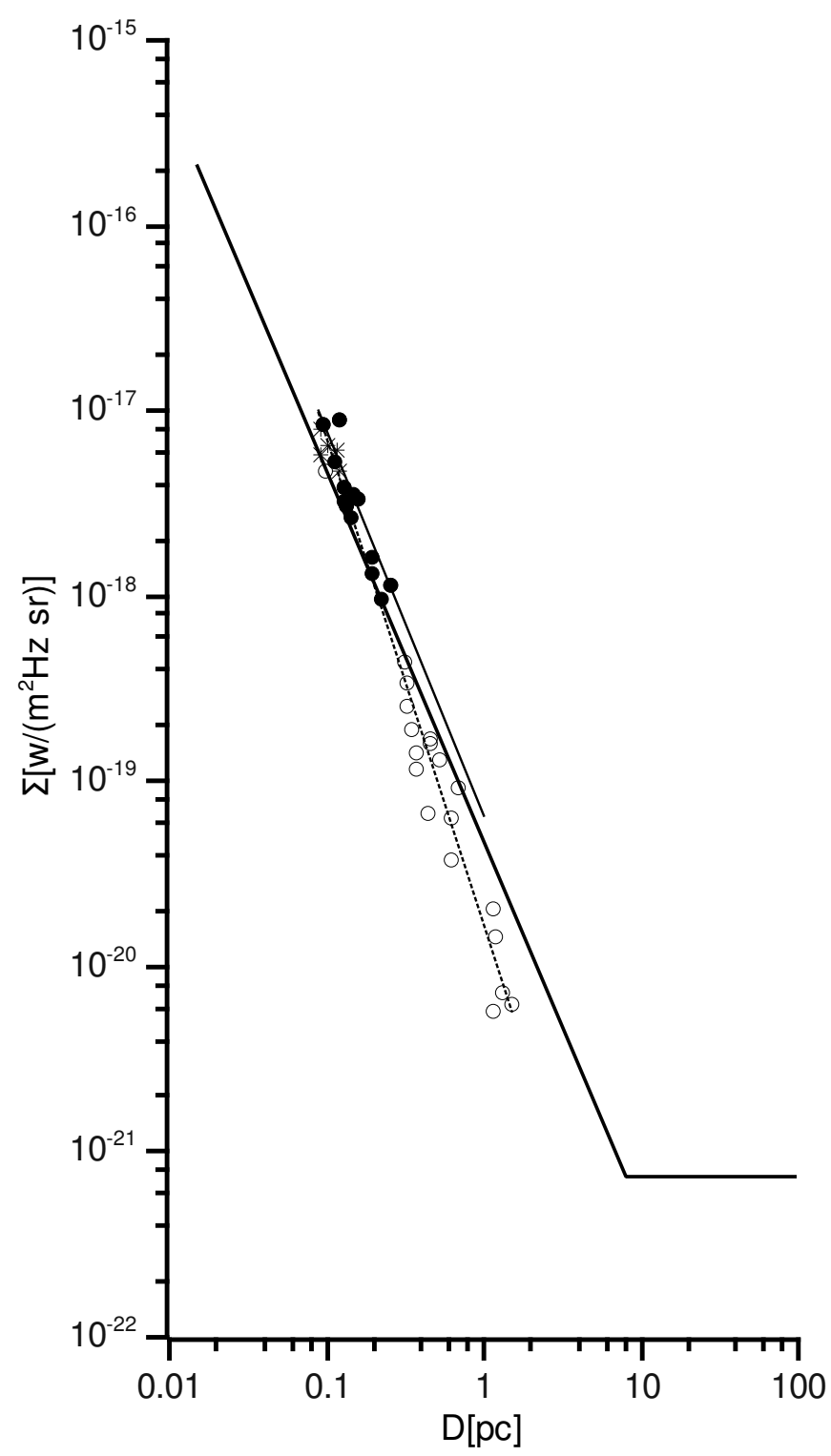

Fig. 2. The Monte Carlo generated sample at $8.64 \mathrm{GHz}$ for a scatter of 2 and simulated slope of -2.7 . The LMC data points (asterisks) are plotted along with the sensitivity (thick solid) line; artificially generated points are plotted above (filled circles) and below (open circles) this line. The selection of points above the sensitivity line give a fit with a flatter slope (thin solid line) than the slope of a fit using all simulated points (dotted line).

Dudziak, G., Péquignot, D., Zijlstra, A. A., \& Walsh, J. R. 2000, A\&A, 363, 717 Filipović, M. D., Cohen, M., Reid, W. A., et al. 2009, MNRAS, in press, [arXiv: 0906.4588v1]

Hilditch, R. W., Howarth, I. D., \& Harries, T. J. 2005, MNRAS, 357, 304

Payne, J. L., Filipovic, M. D., Crawford, E. J., et al. 2008a, Serb. Astron. J., 176, 65

Payne, J. L., Filipovic, M. D., Millar, W. C., et al. 2008b, Serb. Astron. J., 177, 53

Phillips, J. P. 2002, ApJS, 139, 199

Poveda, A., \& Woltjer, L. 1968, AJ, 73, 65

Shklovskii, I. S. 1960, AZh, 37, 256

Stanghellini, L., Shaw, R. A., \& Villaver, E. 2008, ApJ, 689, 194

Urošević, D., Pannuti, T. G., Duric, N., \& Theodorou, A. 2005, A\&A, 435, 437 Urosevic, D., Vukotic, B., Arbutina, B., \& Ilic, D. 2007, Serb. Astron. J., 174, 73 Urošević, D., Vukotić, B., Arbutina, B., Ilić, D., et al. 2009, A\&A, 495, 537 